\title{
Sloan Kettering Virus
}

National Cancer Institute

\section{Source}

National Cancer Institute. Sloan Kettering Virus. NCI Thesaurus. Code C14344.

The Sloan-Kettering viruses (SKVs) are a group of transforming retroviruses that were isolated from chicken embryo cells which had been infected with the avian leukosis virus transformation-defective Bratislava 77 (tdB77). (from Li et al. J Virol 1986;57:1065-72) 